\title{
A Symposium of Large Scale Digital Calculating Machinery
}

Under the joint sponsorship of Harvard University and the Bureau of Ordnance, United States Navy, 336 representatives of university, industrial and government laboratories and research groups met in Cambridge, Mass. from January 7-10, 1947 to participate in a four-day Symposium on Large Scale Digital Calculating Machinery.

The meetings were held in Harvard's new Computation Laboratory and, in part, the Symposium celebrated the formal opening of this new facility.

In addition to the extensive group of technical papers, the program included a demonstration of the Automatic Sequence Controlled Calculator (usually referred to as the Mark I Calculator), and a preview of the Mark II Calculator which is now being assembled at Harvard for the Bureau of Ordnance. The latter machine will later be reassembled at the Dahlgren Proving Ground of the Navy.

The program of the Symposium was as follows:

I. Tuesday, 7 January, 10:00 a.m.

Opening Addresses by

Edward Reynolds, Administrative Vice-President of Harvard University

Rear Admiral C. T. Joy, USN, Naval Proving Ground, Dahlgren, Virginia

Prof. H. H. Aiken, Technical Director of the Computation Laboratory, Harvard University

Inspection of the Computation Laboratory and of Mark II Calculator

II. Tuesday, 7 January, 2:00 p.m.

Existing Calculating Machines, Prof. W. E. Bleick, Chairman

1. "The Work of Charles Babbage" by Mr. R. H. Babbagel

2. "Mark I Calculator" by Mr. R. M. Bloch

3. "Brief description and operating characteristics of the ENIAC" by Dr. L. P. Tabor

4. "Bell Telephone Laboratories relay computing systems" by Mr. S. B. Williams

5. "Mark II Calctilator" by Mr. R. V. D. Campbell

III. Wednesday, 8 January, 9:30 a.m.

The Logic of Large Scale Calculating Machines, Prof. W. H. Furry, Chairman

1. "Problems of mathematical analysis involved in machine computations" by Dr. A. W. Wundheiler

2. "The organization of large scale calculating machinery" by Dr. G. R. Stibitz

IV. Wednesday, 8 January, 2:00 p.m.

Storage Devices, Dr. J. H. Curtiss, Chairman

1. "Mercury delay lines as a memory unit" by Dr. T. K. Sharpless

2. "Slow electromagnetic waves" by Prof. L. N. Brillouin

3. "High Speed electrostatic storage" by Mr. J. W. Forrester

4. "Magnetic and phosphor coated disks" by Dr. B. L. Moore

5. "The selectron-a tube for selective electrostatic storage" by Dr. Jan Rajchman

6. "Optical and photographic storage techniques" by Dr. A. W. Tyler

V. Thursday, 9 January, 9:30 a.m.

Numerical Methods and Suggested Problems for Solution, Dr. Mina S. Rees, Chairman

1. "Method of finite differences for the solution of partial differential equations" by Prof.

Richard Courant 
2. "On computational techniques for certain problems in fluid dynamics" by Dr. R. J. Seeger

3. "Computational problems arising in connection with economic analysis of interindustrial relationships" by Prof. W. W. Leontief

4. "On the accumulation of errors in processes of integration on high speed calculating machines" by Prof. H. A. Rademacher

5. "Fluid mechanics computations" by Prof. H. W. Emmons

6. "Firing tables" by Dr. L. S. Dederick

VI. Thursday, 9 January 2.00 p.m.

Sequencing, Coding, and Problem Preparation, Prof. J. A. Stratton, Chairman

1. "Coding for large scale calculating machinery" by Dr. H. H. Goldstine

2. "Preparation of problems for EDVAC-type machines" by Dr. J. W. Mauchly

3. "The preparation of problems for large scale calculating machinery" by Mr. J. 0. Harrison, Jr.

VII. Friday, 10 January, 9:30 a.m.

Input and Output Devices, Prof. E. L. Chaffee, Chairman

1. "Application of printing telegraph techniques to large scale calculating machinery" by Mr. F. G. Miller

2. "Some physical aspects of magnetic recording": by Mr. Otto Kornei

3. "The numeroscope" by Mr. H. W. Fuller

4. "Input and Output Devices for electronic digital calculating machinery" by Mr. S. N. Alexander

5. "An input device using multiple gates" by Dr. Morris Rubinoff

6. "Photographic methods of handling input and output data" by Dr. K. G. Macleish

7. "Transfer between external and internal memory" by Mr. C. B. Sheppard

VIII. Friday, 10 January, 2:00 p.m.

Conclusions and Open Discussion, Prof. C. C. Bramble, Chairman

1. "Publication, classification, and patents" by Prof. S. H. Caldwell

2. "Le domaine du calcul mécanique" by Dr. Louis Couffignal (paper not read, but to be included in the published report).

3. "New Vistas in mathematics" by Dr. A. T. Waterman

All of the remarks and papers of the 34 speakers, as well as the related discussions, are to be published in a later volume of the Annals of the Computation Laboratory.

Both Harvard University and the Bureau of Ordnance are to be congratulated for their enterprise in making possible this significant meeting. The extensive program reflected every phase of the sweeping progress being made in the field of large-scale calculators, and the large attendance offered a unique opportunity for the numerous small group meetings which do much to facilitate the exchange of helpful information.

The following members registered for the Symposium:

Milton Abramowitz, mathem., NBSMTP

Erik Ackerlind, group leader, Northrop Aircraft Corp., Hawthorne Field, Cal.

L. V. Ahlfors, prof. math., Harvard Univ.

H. H. Aiken, prof. appl, math., Harvard Univ.

L. W. Alberts, grad. stu., Harvard Law Sch.

S. N. Alexander, group leader, computer components group, National Bureau of Standards (NBS)

F. J. Alterman, engin., Reeves Instrument Corp., New York

E. G. Andrews, engin., Bell Tel. Lab., New York

R. C. Archibald, prof. math., Brown Univ. 
George Arfken, Jr., grad. stu., Yale Univ.

K. J. Arnold, assist. prof. math., Univ. Wisconsin

R. H. Babbage, editor, 241 St. James St. West, Montreal, Canada

Rear Admiral W. D. Baker, commandant, U. S. Naval Base, Boston

M. D. Baller, group leader, computers, Army Air Force (AAF), Malden, Mass.

Colin Barnes, prof. physics, Univ. Toronto

Dr. J. L. Barnes, consultant, Raytheon Mfg. Co., Waltham, Mass.

R. M. Barrett, electronic engin., Watson Lab., AAF AMC, Cambridge

Phyllis P. Barrows, res. assist., Harvard Univ.

C. E. Barthel, Jr., electronic computing and control group, Naval Ordnance Lab., Wash.

Walter Bartky, prof. math., Univ. Chicago.

Dr. A. E. Benfield, visiting lect. appl. physics, Harvard Univ.

William Bentinck-Smith, ed. Harvard Alumni Bull., Harvard Univ.

R. J. Bergemann, electronic engin., Office Naval Res., Boston

Dr. Stefan Bergman, res. lect. aeronautical engin., Harvard Univ.

E. C. Berkeley, methods analyst, Prudential Life Ins. Co., Newark, N. J.

A. A. Berlinsky, lab. electrician, Bureau of Census, Wash.

C. H. Berry, prof. mech. engin., Harvard Univ.

J. H. Bigelow, chief engin., Institute Adv. Studies, Princeton, N. J.

Garrett Birkhoff, prof. math., Harvard Univ.

W. E. Bleick, assoc. prof. math., post grad. sch., U. S. Naval Acad., Annapolis, Md.

Daniel Blitz, res. engin., Raytheon Mfg. Co., Waltham, Mass.

R. M. Bloch, res. assist., Harvard Univ.

J. H. Blythe, nautical scientist, Hydrographic Office, Navy Dept., Wash.

Richard Blythe, res. assoc. electr. engin., Mass Inst. Techn.

J. M. Boermeester, actuarial assoc., John Hancock Mutual Life Ins. Co., Boston

B. A. Boley, instr. aeron. engin., Polytechnic Inst., Brooklyn, N. Y.

Capt. C. T. Bonney, prof. naval sci. and tactics, Harvard Univ.

H. R. Boyd, engin., servomechanisms lab., Mass. Inst. Techn.

C. C. Bramble, prof. math., post-grad. sch., U. S. Naval Acad.

Dr. A. E. Brandt, statist., Naval Ordnance Lab., Wash.

Mark Breiter, mathem., office chief ordnance, Pentagon Bldg., Wash.

Ruth A. Brendel, instr. math., Univ. Buffalo

P. W. Bridgman, prof. math. and nat. phil., Harvard Univ.

L. N. Brillouin, prot. appl. math., Harvard Univ.

D. R. Brown, electronic engin., servomechanisms lab., Mass. Inst. Techn.

O. E. Brown, assoc. prof. math., Case Sch. Appl. Sci., Cleveland, O.

R. L. Brown, research assoc., Mass. Inst. Techn.

T. H. Brown, prof. business statistics, Harvard Univ.

Capt. C. A. Buchanan, res. and develop. div., Navy Dept., Bur. Ord., Wash.

Dr. R. S. Burington, chief mathem., evaluation and analysis group, Navy Dept., Bur. Ord., Wash.

S. H. Caldwell, prof. electr, engin., Mass. Inst. Techn.

H. Campaigne, mathem., Office Chief Naval Operations, Wash.

Commander A. A. A. Campbell, Recruiting Station, First Naval District, Boston

R. V. D. Campbell, res. assoc., Harvard Univ.

W. L. Campbell, prof. food techn., Mass. Inst. Techn.

Dr. E. W. Cannon, chief, machinery devel. sect., NBS

Achille Capecelatro, physicist, Underwater Sound Lab., New London, Conn.

F. E. Carey, fellow in journalism, Harvard Univ.

Commander J. H. Carmichael, res. and development div., Navy Dept., Bur. Ord., Wash.

Arthur Casagrande, assoc. prof. civil engin., Harvard Univ.

E. L. Chaffee, prof. physics and commun. engin., Harvard Univ.

Harold Chestnut, engin., Gen. Electr. Co., Schenectady, N. Y.

B. F. Cheydleur, analysis sect., Naval Ord. Lab., Wash. 
Chuan Chu, res. assoc., Univ. Penn.

W. H. Claflin, Jr., treasurer Harvard Coll., 24 Milk St., Boston

G. M. Clemence, director, Naut. Alm. Off., Naval Obs., Wash.

Dr. R. F. Clippinger, mathem., Ballistic Res. Lab., Aberdeen Proving Gr., Md.

Admiral W. T. Cluverius, president, Worcester Polyt. Inst.

Charles Concordia, engin., Gen. Electr. Co., Schenectady, N. Y.

Patrick Conley, teaching fellow appl. physics, Harvard Univ.

J. M. Coombs, engin., Engineering Res. Assoc. Inc., St. Paul, Minn.

Lester Corrsin, grad. stu., Mass. Inst. Techn.

Mary Coulan, admin. assist., Harvard Law School

Richard Courant, prof. math., New York Univ.

Perry Crawford, Jr., director, Spec. Devices Center, Office Naval Res., Port Washington, L. I.

Dr. J. H. Curtiss, assist. to the director, NBS

H. N. Cutler, stu., Harvard Coll.

J. F. Daly, statist., Bureau of Census, Wash.

Dr. G. B. Dantzig, techn. adv. (math.), AAF Office Air Comptr., Pentagon Bldg., Wash.

D. J. Davis, stu., Harvard Coll.

Dr. L. S. Dederick, assoc. director, Ballistic Res. Lab., Aberdeen Proving Gr., Md.

R. L. DeVeer, sales engin., C. P. Clare Co., Boston

Rear Admiral M. L. Deyo, commandant, First Naval Distr., Boston

A. H. Dickinson, engin., Watson Lab., IBM Corp., New York

S. H. Dodd, Jr., res. engin., Mass. Inst. Techn.

Dr. F. W. Dresch, principal mathem., Naval Proving Gr., Va.

B. M. Durfee, engin., IBM Corp., Endicott, N. Y.

Dr. J. J. Eachus, Office Chief Naval Oper., Navy Dept., Wash.

Dr. W. J. Eckert, director pure sci., Watson Lab., IBM Corp., New York

N. E. Edlefsen, engin., Raytheon Mfg. Co., Waltham, Mass.

Murray Ellis, res. assist., Harvard Univ.

G. V. Eltgroth, divisional patent attorney, Bendix Aviation Corp., Baltimore, Md.

H. W. Emmons, assoc. prof. mech. engin., Harvard Univ.

Bernard Epstein, res. assoc. aeronaut. engin., Harvard Univ.

R. R. Everett, res. engin., Mass. Inst. Techn.

Harris Fahnestock, res. engin., Mass. Inst. Techn.

Dr. J. H. Faull, Jr., head sci. sect., Office Naval Res., Boston

Samuel Favor, mech. engin., Computation Lab., Harvard Univ.

H. Feistel, physicist, Watson Lab., AAF AMC, Cambridge, Mass.

L. A. Fine, instr. math., Harvard Univ.

H. W. Flagg, sales repr., Marchant Calc. Machine Co., Boston

D. A. Flanders, assoc. prof. math., New York Univ.

Margaret I. Florencourt, res. engin., servomechanisms lab., Mass. Inst. Techn.

N. C. Ford, assist. ąctuary, Mass. Mutual Life Ins. Co., Springfield, Mass.

J. W. Forrester, assoc. dir., servomechanisms lab., Mass. Inst. Techn.

Donald Foster, physicist, Watson Lab., AAF AMC, Cambridge

L. V. Foster, optical engin., Bausch \& Lomb Co., Rochester, N. Y.

P. G. Frank, lect. physics and math., Harvard Univ.

Stanley Frankel, assist. prof., Univ. Chicago

L. F. Freedman, mech. engin., Reeves Instr. Corp., New York

A. E. Frost, assist. to automatics res. engin., Western Union Tel. Co., New York

H. W. Fuller, res. assist., Harvard Univ.

W. H. Furry, assoc. prof. physics, Harvard Univ.

I. R. Gale, expediter purchaser, Raytheon Mfg. Co., Waltham, Mass.

Herbert Galman, physicist, Exper. Br., Frankford Arsenal, Philadelphia, Pa.

Dr. H. L. Garabedian, prin. physicist, Clinton Labs., Monsanto Chem. Co, Knoxville, Tenn.

C. S. Gardner, stu., Harvard Univ. 
E. B. Gardner, operations evaluations group, Mass. Inst. Techn.

Dr. H. F. Gingerich, mathem., Naval Communi. Annex, Wash.

Capt. J. A. Glick, insp. naval material, 495 Summer St., Boston

R. H. Goddard, develop. engin., Sylvania Electric Products, Inc., Boston

J. A. Goff, dean, Towne Sci. Sch., Univ. Penn.

Dr. H. E. Goheen, mathem., Office Naval Res., Boston

Dr. H. H. Goldstine, res. fellow, Institute Adv. Studies, Princeton, N. J.

Harold Grad, grad. stu., New York Univ.

R. B. Graham, chief engin. SPDE, Bendix Aviation Corp., Teterboro, N. J.

J. V. Granger, grad. stu., Harvard Univ.

E. F. Grant, radio engin., Watson Lab., AAF AMC, Cambridge

Serge Gratch, instr. mech. engin., Univ. Penn.

H. V. Greenough, Jr., director, Technichord Records, Cambridge

R. H. Griest, res. engin., Hughes Aircraft Corp., Culver City, Cal.

W. C. Griffith, teaching fellow engin. sci., Harvard Univ.

S. E. Gudmundson, civil engin., Aeronaut. Res. Inst. of Sweden, Ulvsunde, Sweden

G. Hagg, prof., Univ. Upsala, Sweden

B. Hamilton, superv. IBM, Mass. Gen. Hosp., Boston

F. E. Hamilton, engin., IBM Corp., Endicott, N. Y.

Dr. R. W. Hamming, engin., Bell Tel. Labs., Murray Hill, N. J.

E. B. Hammond, Jr., res. engin., Sperry Gyroscope Co., Great Neck, L. I.

M. H. Hansen, statist. assist. to director, Bureau of Census, Wash.

J. O. Harrison, Jr., res. assoc., Harvard Univ.

R. W. Hart, electr. engin., Office Naval Res., Boston

Bernhard Haurwitz, assoc. prof. meteorology, Mass. Inst. Techn.

Capt. E. C. Hawk, off. superv. insp. naval material, First Naval District, Boston

R. L. Hawkins, chief technician, Computation Lab., Harvard Univ.

H. L. Hazen, prof. electr. engin., Mass. Inst. Techn.

Dr. R. W. Hickman, lect. appl. physics, Harvard Univ.

G. W. Hobbs, electronics engin., Gen. Electric Co., Schenectady, N. Y.

J. V. Holberton, Ballistics Res. Lab., Aberdeen Proving Gr., Md.

L. M. Hollingsworth, chief components lab., Watson Lab., AAF AMC, Cambridge

R. E. Hopkins, assoc. prof., Univ. Rochester

Dr. Grace M. Hopper, res. fellow, Harvard Univ.

A. B. Horton, Jr., res. assist. electr. engin., Mass. Inst. Techn.

Dr. A. S. Householder, prin. physicist, Clinton Laboratories, Oak Ridge, Tenn.

J. H. Howard, director lab., Engineering Res. Associates Inc., St. Paul, Minn.

B. C. Howland, reporter, Boston Post

E. S. Hughes, Jr., designer, IBM Corp., Endicott, N. Y.

F. B. Hunt, jun. engin., N. E. Power Service Co., Boston

F. V. Hunt, assoc. prof. appl. physics, Harvard Univ.

Dr. J. M. Ide, techn. director, Underwater Sound Lab., New London, Conn.

Fritz John, assoc. prof. appl. math., New York Univ.

Rear Admiral C. T. Joy, commanding officer, Naval Proving Gr., Dahlgren, Va.

H. R. Kaiser, res. engin., Univ. Calif., Los Angeles

G. N. Kamm, grad. stu., Harvard Univ.

Martin Katzin, consultant, Naval Res. Lab., Wash.

R. Kelner, stu., Harvard Univ.

Constance Kepke, res. assist., Harvard Univ.

Capt. C. H. J. Keppler, counsellor foreign students, Harvard Univ.

Dr. R. B. Kershner, appl. physics lab., The Johns Hopkins Univ.

Dr. G. W. King, res. chemist, Arthur D. Little Inc., Cambridge

R. W. P. King, assoc. prof. appl. physics, Harvard Univ.

Bertram Klein, res. assoc., Polytechnic Inst., Brooklyn, N. Y.

Ruth A. Knowlton, secretary, Harvard Univ. 
Dr. Zdenek Kopal, res. assoc. eléctr. engin., Mass. Inst. Techn.

Otto Kornei, res. engin., The Brush Development Co., Cleveland, 0.

Capt. W. E. Kraft, superv. inspec. naval material, First Naval District, Boston

George Kramer, reporter, The Harvard Crimson

J. A. Krumhansl, assist. prof. physics, Brown Univ.

Jack Laderman, mathem., NBSMTP

Emory Lakatos, engin., Bell Tel. Labs., Murray Hill, N. J.

C. D. Lake, development engin., IBM Corp., Endicott, N. Y.

Dr. O. E. Lancaster, mathem., Bureau Aeronautics, Navy Dept., Wash.

Dr. Philippe LeCorbeiller, lect. appl. physics, Harvard Univ.

Dr. Joseph Lehner, mathem., Hydrocarbon Research Inc., New York

Alfred Leitner, res. assist., Yale Univ.

W. W. Leontief, prof. economics, Harvard Univ.

Dr. A. A. Levin, physicist, Bausch \& Lomb Optical Co., Rochester, N. Y.

Dr. J. H. Levin, chief machines sect. computing lab., Ballistic Res. Lab., Aberdeen Proving Gd., Md.

M. L. Libman, patent attorney, Office Chief Ord., War Dept., Wash.

Dr. D. M. Little, secy. to univ., Harvard Univ.

Barbara Livingston, secy. to dean, Harvard Law School

W. S. Loud, res. engin., Mass. Inst. Techn.

Samuel Lubkin, engin., Ballistic Res. Lab., Aberdeen Proving Gd., Md.

Herman Lukoff, res. assist., Univ. Penn.

J. R. Macdonald, res. engin., Mass. Institute Techn.

Capt. Louis McGlone, director training, First Naval District, Boston

G. W. Mackey, assist. prof. math., Harvard Univ.

Saunders MacLane, prof. math., Harvard Univ.

Dr. K. G. Macleish, physicist, Eastman Kodak Co., Rochester, N. Y.

A. B. Macnee, res. assist. electr. engin., Mass. Inst. Techn.

Kathleen McNulty, Ballistic Res. Lab., Moore Sch., Univ. Penn.

J. L. McPherson, statistician, Bureau of Census, Wash.

C. Malik, electronic engin., Watson Lab., AFC AMC, Cambridge

R. A. Mangini, manager planning dept., John Hancock Mutual Life Ins. Co., Boston

Charles Manneback, prof. theoretical physics, Univ. Louvain

L. C. Mansur, radar engin., Watson Lab., AAF AMC, Cambridge

T. M. Marcus, NRC fellow, Mass. Inst. Techn.

L. J. Marsee, planning engin., Mass. Mutual Life Ins. Co., Springfield, Mass.

R. C. Martin, Jr., stu., Harvard Univ.

A. H. Mathewson, purchasing agent, Mass. Gen. Hospital, Boston

Dr. J. W. Mauchly, Electronic Control Co., 1215 Walnut St., Philadelphia, Penn.

Margaret W. Mayall, res. assist., Harvard Coll. Observatory, Cambridge

D. H. Menzel, prof. astrophysics, Harvard Coll. Observatory, Cambridge

Nicholas Metropolis, assist. prof., Univ. Chicago

F. A. Metz, electronic engin., Watson Lab., AAF AMC, Cambridge

J. W. Miles, physicist, Univ. Calif., Los Angeles

F. G. Miller, electrical engin., Computation'Lab., Harvard Univ. and Dahlgren Proving

Gr., Dahlgren, Va.

H. R. Mimno, prof. appl. physics, Harvard Univ.

Richard von Mises, prof. aerodynamics and appl. math., Harvard Univ.

Herbert Mitchell, grad. stu., Computation Lab., Harvard Univ.

E. B. Mode, prof. math., Boston Univ., Boston

R. J. Monroe, assist. director, Inst. Statistics, Univ. N. Carolina, Raleigh, N. C.

C. N. Mooers, stu. reporter, Mass. Inst. Techn.

Dr. B. L. Moore, res. fellow. Harvard Univ.

Commander H. M. Mott-Smith, res. and develop. div., Navy Dept., Bur. Ord., Wash. 
Dr. C. F. Muckenhoupt, assist. chief sci. center, Office Naval Res., Boston

G. G. Muller, engin., Bell Tel. Labs., Murray Hill, N. J.

H. E. Nash, physicist, Underwater Sound Lab., New London, Conn.

R. B. Neal, project engin., Sperry Gyroscope Co., Great Neck., L. I.

H. H. Newell, prof. electr. engin., Worcester Polyt. Inst.

H. B. Nichols, science ed., Christian Sci. Monitor, Boston

Natalie N. Nicholson, libr., Grad. Sch. Engin., Harvard Univ.

Dr. E. N. Nilson, analytical engin., United Aircraft .Corp., East Hartford, Conn.

Capt. K. H. Noble, assist. chief res. and development, Navy Dept., Bur. Ord., Wash.

W. J. Nolan, Jr., res. assoc. electr. engin., Mass. Inst. Techn.

Dr. Harry Nyquist, engin., Bell Tel. Lab., New York

J. A. O'Brien, res. assist. electr. engin., Mass. Inst. Techn.

J. W. O'Connor, office of vice-pres., John Hancock Mutual Life Ins. Co., Boston

Otto Oldenberg, prof. physics, Harvard Univ.

B. M. Oliver, engin., Bell Tel. Lab., New York

R. D. O'Neal, physicist, Eastman Kodak Co., Rochester, N. Y.

I. B. Parkhurst, assist. business manager, Harvard Coll.

Dr. C. L. Pekeris, IBM Corp., Watson Lab., Columbia Univ.

Lt. Comdr. J. T. Pendergrass, Office Chief Naval Operations, Navy Dept., Wash.

C. G. Peterson, agent, Marchant Calculating Machine Co., Boston

J. H. Phillips, British Supply Office, 1785 Mass. Av., N. W., Wash.

Dr. E. R. Piore, electronic sec., Office Naval Res., War Dept., Wash.

Dr. L. A. Pipes, res. physicist, Hughes Aircraft Co., Culver City, Cal.

G. P. Ploetz, electronic engin., Watson Lab., AAF AMC, Cambridge

W. A. Porter, constr. supervisor, Harvard Univ.

H. A. Rademacher, prof. math., Univ. Penn.

Dr. Jan Rajchman, res. physicist, Radio Corp. Amer. Labs., Princeton, N. J.

E. A. Rasor, actuarial mathem., Social Security Admin., Wash.

I. G. Recant, aeronautical engin., Nat. Adv. Comm. Aeronautics, Langley Field, Va.

Dr. Mina S. Rees, chief, math. sect., Office Naval Res., Wash.

D. K. Reynolds, electronic res. lab., Cruft Lab., Harvard Univ.

Edward Reynolds, administrative vice-pres., Harvard Univ.

George Reynolds, engin. aide, Watson Lab., AAF AMC, Cambridge

C. H. Richards, electrr. designer, Harvard Univ.

Dr. F. D. Rigby, mathem., Navy Dept., Office Naval Res., Wash.

R. F. Rinehart, prof. math., Case Sch. Appl. Sci., Cleveland, 0.

J. W. Roche, technician, Computation Lab., Harvard Univ.

E. Rodriguez, stu., Harvard Coll.

Commander J. B. Roman, material office for personnel, First Naval District, Boston

J. H. Rosenbloom, physicist, Frankford Arsenal, Philadelphia

Dr. Morris Rubinoff, instr. physics, Harvard Univ.

Reinhold Rudenberg, prof. electr. engin., Harvard Univ.

J. B. Russell, assoc. prof. electr. engin., Columbia Univ.

E. A. Ryavec, mathem., Navy Dept., Office Naval Res., Wash.

R. C. Sandborn, stu., Harvard Coll.

B. L. Sarahan, mathem., Naval Res. Lab., Anacostia, Wash.

Lt. Comdr. W. L. Savidge, distr. in telligence officer, First Naval District, Boston

J. B. Scarborough, prof. math., U. S. Naval Acad., Annapolis, Md.

A. C. Schaeffer, assoc. prof. math., Stanford Univ.

F. F. Schaller, jun. engin., N. E. Power Service Co., Boston

T. E. Schuette, project engin., Barber Colman Co., Rockford, Ill.

R. E. Scott, res. assist. electr. engin., Mass. Inst. Techn.

H. E. Seaton, electr. engin., The Autocall Co., Shelby, O.

R. R. Seeber, Jr., res. assist., Watson Lab., IBM Corp., New York 
Dr. R. J. Seeger, physicist, chief. mech. div., Naval Ordnance Lab., White Oaks, Silver Springs, Md.

Dr. Harner Selvidge, director special products dev., Bendix Aviation Corp., Teterboro, N. J.

Dr. C. E. Shannon, engin., Bell Tel. Lab., New York

Dr. T. K. Sharpless, project super. EDVAC, Univ. Penn.

C. B. Sheppard, Electronic Control Co., 1215 Walnut St., Philadelphia

Martin Shwartz, mathem., Office Naval Res., Boston

L. B. Slichter, prof. geophysics, Univ. Wisconsin

A. E. Smith, mathem., Navy Dept., Office Naval Res., Washington

Dr. C. L. V. Smith, senior engin., Raytheon Mfg. Co., Waltham, Mass.

V. G. Smith, prof. electr. engin., Univ. Toronto

Frances E. Snyder, mathem., ENIAC, Ballistic Res. Lab., Aberdeen Proving Gr., Md.

Andrew Sobczyk, mathem., Watson Lab., AAF AMC, Cambridge

R. A. Spence, engin., office bldgs. and grounds, Harvard Univ.

Dr. R. C. Spencer, chief, antenna lab., Watson Lab., AAF AMC, Cambridge

L. R. Steinhardt, director sp. projects, Engineering Res. Associates Inc., St. Paul, Minn.

Dr. T. E. Sterne, chief ballistician, Ballistic Res. Lab., Aberdeen Proving Gr., Md.

Dr. G. R. Stibitz, consultant appl. math., 393 South Prospect St., Burlington, Vt.

Dr. W. W. Stifler, Jr., physicist, Underwater Sound Lab., New London, Conn.

J. A. Stratton, director, Res. Lab. Electronics, Mass. Inst. Techn.

Dr. L. P. Tabor, chief electronics sect., Moore Sch., Univ. Penn.

G. R. Tatum, assoc. director res. appl. physics, The Johns Hopkins Univ.

Margaret O. Taylor, mathem., Gulf Res. and Development Co., Pittsburgh, Pa.

Richard Taylor, assist. prof. electr. engin., Mass. Inst. Techn.

L. H. Thomas, physicist, IBM Corp., New York

Lt. P. D. Thompson, AAF, res. assist., Institute Adv. Studies, Princeton, N. J.

Capt. F. P. Tibbits, ordnance officer, Charlestown Naval Base, Boston

$P$. D. Tilton, res. assist. electr. engin., Mass. Inst. Techn.

Dr. C. B. Tompkins, engin., Engineering Res. Associates Inc., St. Paul, Minn.

Dr. A. M. Turing, research fellow, National Res. Lab., Teddington, England

Dr. A. W. Tyler, physicist, Eastman Kodak Co., Rochester, N. Y.

C. F. Van Aken, chief machine tabul. div., Bureau of Census, Washington

Capt. A. M. Van Eaton, commanding officer, Office Naval Res., Boston

F. M. Verzuh, res. assoc. electr. engin., Mass. Inst. Techn.

J. H. Wakelin, res. supervisor, Engineering Res. Associates Inc., St. Paul, Minn.

Henry Wallman, assoc. prof. math., Mass. Inst. Techn.

John Walsh, electr. engin., Watson Lab., AAF AMC, Cambridge

J. H. Warden, president, The Autocall Co., Shelby, 0.

K. L. Warthman, engin., Bell Tel. Lab., New York

Dr. A. T. Waterman, chief sci. planning div., Navy Dept., Office Naval Res., Wash.

J. R. Weiner, engin., Raytheon Mfg. Co., Waltham, Mass.

Albert Wertheimer, head engin., Navy Dept., Bur. Ord., Washington

Dr. F. J. Weyl, mathem., Navy Dept., Bur. Ord., Wash.

D. P. Wheatland, Cruft Lab., Harvard Univ.

Stephen White, science news writer, New York Herald-Tribune

Lt. Comdr. A. W. Whitney, aide to commandant, First Naval District, Boston

Hassler Whitney, prof. math., Harvard Univ.

D. V. Widder, prof. math., Harvard Univ.

Maria Wilhelm, science sect., Newsweek Mag., New York

R. E. Wilkins, mech. engin., Harvard Univ.

S. B. Williams, cons. electr. engin., 366 Clermont Ave., Brooklyn, N. Y.

Capt. J. H. Willingham, Jr., commandant, Naval Ammunition Depot, Hingham, Mass.

M. K. Wood, techn. assist., progr. monitoring div., headquarters, Army Air Force, Wash.

L. F. Woodruff, techn. chief sci. branch, W.D.G.S. 2, Pentagon Bldg., Wash. 
R. L. Wooley, electronic engin., Watson Lab., AAF AMC, Cambridge

Dr. A. W. Wundheiler, mathem., Navy Dept., Bur. Ord., Wash.

Jeff Wylie, bureau head, Boston Office, Time Mag.

Patrick Youtz, res. assoc. electr. engin., Mass. Inst. Techn.

Dr. V. K. Zworykin, director electronic res., Radio Corp. Amer., Princeton, N. J.

S. H. C.

1 Richard Henry Babbage (1883- ) is a son of Henry Whitmore B. (1855-1911), son of Henry Prevost B. (1824-1918), son of Charles Babbage (1792-1871). He is an assistant editor of Canada's national farm magazine, Family Herald and Weekly Star.

\section{APPENDIX}

A. Existing and Practically completed Digital Computing Machines developed by or presently on loan or contract to the Government.

I. Department: War

Branch or Bureau: Ordnance Dept.

Permanent Location of Machine: Ballistic Research Lab., Aberdeen Proving Ground, Md.

Type: A. Relay (small) manufactured by IBM Corp.

B. Relay (small) manufactured by IBM Corp.

C. Electronic (ENIAC) manufactured by Moore School of Electrical Engineering, University of Pennsylvania. See $M T A C$, v. 2, p. 97 f.

D. Relay (large) manufactured by Bell Telephone Labs.

II. Department: War

Branch or Bureau: Army Ground Forces

Permanent Location of Machine: Army Ground Forces, no. 4, Fort Bliss, Texas.

Type: Relay (small) manufactured by Bell Telephone Labs.

III. Department: Navy

Branch or Bureau: Office of Naval Research

Permanent Location of Machine: Naval Research Lab., Bellevue, Md.

Type: Relay (small) manufactured by Bell Telephone Labs.

IV. Department: Navy

Branch or Bureau: Bureau of Ordnance

Permanent Location of Machine: Harvard Computation Lab., Cambridge, Mass.

Type: Electromechanical (IBM Sequence Controlled Calculator = "Mark I Calculator"). See $M T A C$, v. 2, p. $185 f$.

V. Department: Navy

Branch or Bureau: Bureau of Ordnance

Permanent Location of Machine: Navy Proving Ground, Dahlgren, Va. [In probable operation by July 1947].

Type: A. Relay (small) manufactured by IBM Corp.

B. Relay (large)"Mark II Calculator," manufactured by the Computation Lab., Harvard Univ. [EDITORIAL Note: It is about 12 times faster than Mark I, and cost about $\$ 400000$.]

VI. Department: National Advisory Committee for Aeronautics

Permanent Location of Machine: Langley Memorial Lab., Langley Field, Va.

Type: Relay, manufactured by Bell Telephone Labs. (under contract expiring in 1948).

B. Present Automatic Digital Computing Machine Construction and Development Projects supported entirely or in part by the Government. 
I. Department: War

Branch or Bureau: Ordnance Department

Description of activity:

A. Moore School of Electrical Engineering, Construction of an electronic digital machine ("EDVAC").

B. Institute for Advanced Study and RCA Labs., Princeton, N. J. Construction of an electronic digital machine (financed only in part by federal funds).

C. National Bureau of Standards, Washington, D. C., Long-range component development program.

II. Department: Navy

Branch or Bureau: Office of Naval Research

Description of Activity:

A. National Bureau of Standards, Construction of an electronic digital machine.

B. Servomechanisms Lab., Mass. Institute of Technology, Construction of an electronic digital machine to be used in a large guided-missile flight simulator.

III. Department: Navy

Branch or Bureau: Bureau of Ordnance

Description of Activity:

A. Harvard Computation Lab., Research and preparation of specifications for an electronic digital machine.

B. Naval Ordnance Lab., White Oaks, Md., Construction of an electronic digital machine, temporarily abandoned (Dec. 1946).

IV. Department: Commerce

Branch or Bureau: Bureau of the Census

Description of Activity: National Bureau of Standards, Construction of an electronic digital machine.

National Bureau of Standards

J. H. Curtiss

Editorial Note: Other projected digital machines, unaided in their construction by the U. S. Government, are being built by:

(i) The Eastman Kodak Co., Rochester, N. Y.

(ii) The University of California, Berkeley.

(iii) The National Physical Laboratory, Teddington, England, under the direction of Dr. Alan M. TURING. The planned Automatic Computing Engine will work at the speed of the ENIAC or possibly somewhat higher, and will take advantage of new technical developments, making possible both a greater memory capacity and a higher degree of complexity in the instructions.

\section{P. G. Scheutz, Publicist, Author, Scientific Mechanician, and Edvard Scheutz, En- gineer,-Biography and Bibliography}

Pehr Georg Scheutz (1785-1873), son of a tavern-keeper, after passing examinations in law, practised in different localities before settling in Stockholm in 1812. In 1817 as owner of a printing establishment founded by the well-known writer F. CEDERBORGH, Scheutz devoted himself from that time principally to literary interests. He soon became part owner and co-editor of a newspaper which, with changed name Argus (1820-1836), became Sweden's most important political newspaper in the 1820's. During 1826- 